Related content

\section{Explaining a changeover from normal to super diffusion in time-dependent billiards}

To cite this article: Matheus Hansen et al 2018 EPL 12160003

View the article online for updates and enhancements. edpsciences IOP Institute of Physics 


\title{
Explaining a changeover from normal to super diffusion in time-dependent billiards
}

\author{
Matheus Hansen ${ }^{1}$, David Ciro $^{2}$, Iberê L. Caldas ${ }^{1}$ and Edson D. Leonel ${ }^{3}$ \\ 1 Instituto de Fúsica, Universidade de São Paulo - São Paulo - CEP 05314-970 SP, Brazil \\ 2 Instituto de Física, Universidade Federal do Paraná - Curitiba - CEP 81531-990 PR, Brazil \\ 3 Departamento de Física, UNESP - Rio Claro - CEP 13506-900 SP, Brazil
}

received 29 January 2018; accepted in final form 25 April 2018

published online 17 May 2018

PACS 05.45.-a - Nonlinear dynamics and chaos

PACS $05.45 . \mathrm{Pq}$ - Numerical simulations of chaotic systems

PACS 05.40.Fb - Random walks and Levy flights

\begin{abstract}
The changeover from normal to super diffusion in time-dependent billiards is explained analytically. The unlimited energy growth for an ensemble of bouncing particles in time-dependent billiards is obtained by means of a two-dimensional mapping of the first and second moments of the speed distribution function. We prove that, for low initial speeds the average speed of the ensemble grows with exponent $\sim 1 / 2$ of the number of collisions with the boundary, therefore exhibiting normal diffusion. Eventually, this regime changes to a faster growth characterized by an exponent $\sim 1$ corresponding to super diffusion. For larger initial energies, the temporary symmetry in the diffusion of speeds explains an initial plateau of the average speed.
\end{abstract}

Copyright (C) EPLA, 2018

As coined by Enrico Fermi [1] Fermi acceleration (FA) is a phenomenon where an ensemble of classical and noninteracting particles acquires energy from repeated elastic collisions with a rigid and time-varying boundary. It is typically observed in billiards [2-4] whose boundaries are moving in time [5-9]. If the motion of the boundary is random and the initial particle speeds are small (compared to the moving boundary), the average speed grows at a rate proportional to $n^{1 / 2}$, with $n$ denoting the number of collisions. If the initial speeds are large, a transient plateau in their average is observed in a plot $\bar{V} v s . n$. This is due to the symmetric diffusion of the speeds to values above and below the initial value [10]. This simmetry is, however, broken when the distribution of speeds reaches a lower bound forcing the mean speed to exhibit a sustained growth.

For deterministic oscillations of the border, the scenario is different. Breathing oscillations preserve the shape but not the area of the billiard. It is known that the average speed evolves in a sub-diffusive manner with exponent of the order $1 / 6[11,12]$. On the other hand, for oscillations preserving the area but not the shape of the billiard there are two regimes of growth. For short times the diffusion of speeds is normal, with growth exponent $\sim 1 / 2$, then, after a sufficiently large number of collisions, it enters to a super diffusion regime, with exponent $\sim 1$ [13]. This changeover is, so far, not yet explained and our contribution in this letter is to fill up this gap in the theory. This is achieved by studying the momenta of the speed distribution function, noticing that the dynamical angular/time variables have an inhomogeneous distribution in phase space.

The results presented in this letter are illustrated by a time-dependent oval-billiard [3] whose phase space is mixed when the boundary is static. The boundary of the billiard is written as $R_{b}(\theta, t)=1+\epsilon[1+a \cos (t)] \cos (p \theta)$, where $R_{b}$ is the radius of the boundary in polar coordinates, $\theta$ is the polar angle, $\epsilon$ controls the circle deformation, $p>0$ is an integer number determining the shape of the boundary ${ }^{1}, t$ is the time and $a$ is the amplitude of the boundary oscillation. Figure 1 shows a typical scenario of the boundary and three subsequent collisions illustrating the dynamics.

The dynamics of the particle is given in terms of a discrete, nonlinear four-dimensional mapping $A: R^{4} \rightarrow R^{4}$, that transforms the dynamical variables at collision $n$ to their new values at collision $n+1,\left(\theta_{n+1}, \alpha_{n+1}, V_{n+1}\right.$, $\left.t_{n+1}\right)=A\left(\theta_{n}, \alpha_{n}, V_{n}, t_{n}\right)$, where $V_{n}=\left|\vec{V}_{n}\right|$ denotes the magnitude of the particle velocity after collision $n$, and $\alpha_{n}$ corresponds to the angle between the particle

\footnotetext{
${ }^{1}$ Non-integer numbers produce open billiard leading to escape of particle through hole on the border.
} 


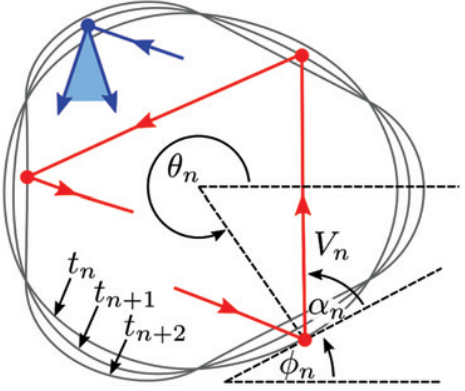

Fig. 1: (Color online) Three consecutive collisions of a particle in a deterministic time time-dependent billiard (red trajectory). Illustration of the reflection angle range for a billiard with random motion in the boundary (blue). The relevant angles and velocity are shown for the $n$-th collision.

trajectory and the tangent line at the $n$-th collision with the boundary at the polar angle $\theta_{n}$ and collision time $t_{n}$ (see fig. 1). Given that each particle moves along a straight line between collisions and with constant speed, the radial position of the particle is given by $R_{p}(t)=$ $\sqrt{X^{2}(t)+Y^{2}(t)}$, where $X(t)$ and $Y(t)$ are the rectangular coordinates of the particle at the time $t$. The angular position $\theta_{n+1}$ is obtained by the numerical solution of $R_{p}\left(\theta_{n+1}, t_{n+1}\right)=R_{b}\left(\theta_{n+1}, t_{n+1}\right)$. The time at collision $n+1$ is given by $t_{n+1}=t_{n}+\sqrt{\Delta X^{2}+\Delta Y^{2}} /\left|\vec{V}_{n}\right|$, where $\Delta X=X_{p}\left(\theta_{n+1}, t_{n+1}\right)-X\left(\theta_{n}, t_{n}\right)$ and $\Delta Y=$ $Y_{p}\left(\theta_{n+1}, t_{n+1}\right)-Y\left(\theta_{n}, t_{n}\right)$. The conservation of momentum law is applied in a non-inertial frame where the boundary is at rest. The reflection laws at the instant of collision are $\vec{V}_{n+1}^{\prime} \cdot \vec{T}_{n+1}=\xi \vec{V}_{n}^{\prime} \cdot \vec{T}_{n+1}$, and $\vec{V}_{n+1}^{\prime}$. $\vec{N}_{n+1}=-\kappa \vec{V}_{n}^{\prime} \cdot \vec{N}_{n+1}$, where the unit tangent and normal vectors are $\vec{T}_{n+1}=\cos \left(\phi_{n+1}\right) \hat{i}+\sin \left(\phi_{n+1}\right) \hat{j}, \vec{N}_{n+1}=$ $-\sin \left(\phi_{n+1}\right) \hat{i}+\cos \left(\phi_{n+1}\right) \hat{j}, \phi=\arctan \left(Y^{\prime}(t) / X^{\prime}(t)\right)$ with $Y^{\prime}(t)=\mathrm{d} Y / \mathrm{d} \theta, X^{\prime}(t)=\mathrm{d} X / \mathrm{d} \theta$ and $\xi, \kappa \in[0,1]$ are the restitution coefficients for the tangent and normal directions. The term $\overrightarrow{V^{\prime}}$ corresponds the velocity of the particle measured in the non-inertial reference frame. The tangential and normal components of the velocity after collision $n+1$ are

$$
\begin{aligned}
\vec{V}_{n+1} \cdot \vec{T}_{n+1} & =\xi \vec{V}_{n} \cdot \vec{T}_{n+1}+(1-\xi) \vec{V}_{b} \cdot \vec{T}_{n+1}, \\
\vec{V}_{n+1} \cdot \vec{N}_{n+1} & =-\kappa \vec{V}_{n} \cdot \vec{N}_{n+1}+(1+\kappa) \vec{V}_{b} \cdot \vec{N}_{n+1},
\end{aligned}
$$

where $\vec{V}_{b}\left(t_{n+1}\right)=\left.\frac{\mathrm{d} R_{b}(t)}{\mathrm{d} t}\right|_{t_{n+1}}\left[\cos \left(\theta_{n+1}\right) \widehat{i}+\sin \left(\theta_{n+1}\right) \widehat{j}\right]$ is the velocity of the moving boundary at time $t_{n+1}$. The speed of the particle after collision $n+1$ is given by $V_{n+1}=$ $\sqrt{\left(\vec{V}_{n+1} \cdot \vec{T}_{n+1}\right)^{2}+\left(\vec{V}_{n+1} \cdot \vec{N}_{n+1}\right)^{2}}$, while the angle $\alpha_{n+1}$ is written as $\alpha_{n+1}=\arctan \left[\frac{\vec{V}_{n+1} \cdot \vec{N}_{n+1}}{\vec{V}_{n+1} \cdot \vec{T}_{n+1}}\right]$.

Given an initial condition $\left(\theta_{n}, \alpha_{n}, V_{n}, t_{n}\right)$, the dynamical properties of the system can be obtained through application of the previous equations. We are interested in the behavior of the average speed, obtained from two different kinds of average, namely $\bar{V}=\frac{1}{M} \sum_{i=1}^{M} \frac{1}{n+1} \sum_{j=0}^{n}|\vec{V}|_{i, j}$, where the first summation is made over an ensemble of

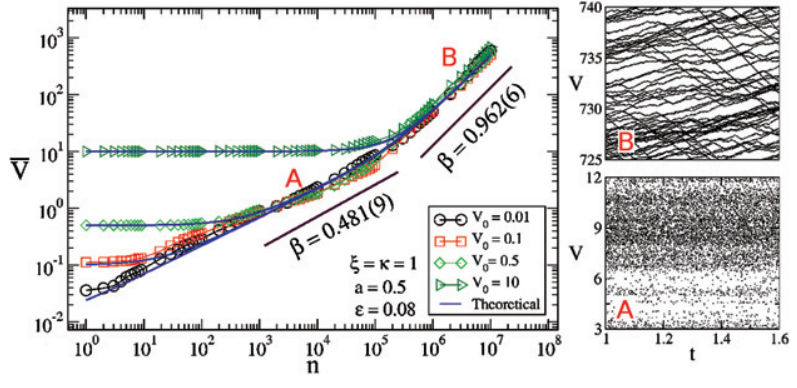

Fig. 2: (Color online) Plot of $\bar{V} v s . n$ for different initial speeds, as labeled in the figure. Three different regimes are clear in the figure. For large initial speeds, a plateau dominates the dynamics for short $n$. After a first crossover, the average speed starts to grow as a power law diffusing the speed as a normal diffusion with slope of $0.481(9)$. Soon after, there is a second crossover where the normal diffusion is replaced by a super diffusion with slope $0.962(6)$. The right panels show portions of a single realization in the $(V, t)$-space where $A$ identifies normal diffusion and $B$ super diffusion. The parameters used are $\epsilon=$ $0.08, a=0.5$ and $p=3$.

different initial conditions randomly distributed in $t \in$ $[0,2 \pi], \alpha \in[0, \pi]$ and $\theta \in[0,2 \pi]$ for a fixed initial speed while the second summation corresponds to an average made over the orbit, hence in time. We considered $M=$ 5000 and $n=10^{7}$ collisions. Figure 2 shows the behavior of $\bar{V}$ vs. $n$ for different initial speeds. Three different kinds of behavior can be seen from the figure. If the initial speed is large, the curve of average speed exhibits a plateau. The size of the plateau depends on the value of the initial speed (see ref. [10] for a discussion of such kind of behavior in a two-dimensional mapping). A higher initial speed, leads to a longer plateau. A first crossover is observed changing the behavior of the constant regime to the regime of growth with a slope of growth typical of normal diffusion. A numerical fitting gives a slope $0.481(9)$. The regime of normal diffusion then reaches a second crossover passing to a faster regime of growth named as super diffusion, with slope $0.962(6)$. The panels on the right-hand side of fig. 2 , give the plot of a single realization in the $(V, t)$ space where $A$ corresponds to normal diffusion and $B$ to super diffusion. We emphasize when the perturbation on the boundary is random, the dynamics in the $(V, t)$-space is similar to what is observed in $A$ with a constant slope of growth about $1 / 2$ therefore characterized by normal diffusion. When the restitution coefficients $\xi, \kappa \neq 1$, inelastic collisions occur leading to a different scenario [14], where the energy growth is interrupted by the violation of Liouville's theorem and attractors emerge in the phase space.

Provided that all particles start with the same speed at a random location and direction they can gain or lose energy upon collisions with the moving boundary and follow very different paths. At a given collision of the full ensemble with the wall, there is roughly the same number of particles gaining and losing speed, then, the mean speed will not change much after the collision [14]. 


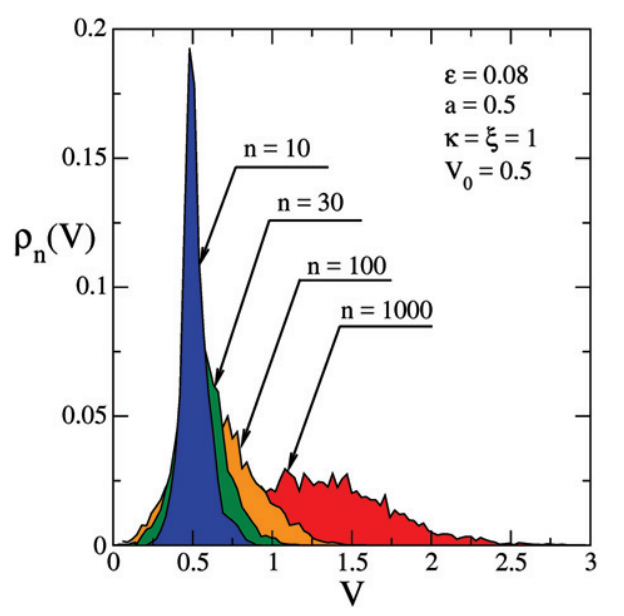

Fig. 3: (Color online) Plot of the evolution of the speed distribution function $\rho_{n}(V)$ for an ensemble of 5000 particles after different number of collisions. The parameters used are $\epsilon=0.08, a=0.5$ and $p=3$.

However, this process, can not continue indefinitely, for there is a lower bound of the speed, i.e., zero, and no upper bound. Particles reaching the lower bound can only gain speed upon collision with the wall. When a sufficient number of particles reaches the lower bound, the symmetry of the speed diffusion is broken and we have a crossover between the constant regime and the growth regime.

Figure 3 shows the evolution of the speed distribution function $\rho_{n}(V)$, for an ensemble of particles as the number of collisions increases. Notice that, after ten collisions the distribution has not reached the lower bound and the mean speed is essentially the initial value of $V_{0}$. This changes after the lower bound is reached, leading the mean to grow as observed after 100 and 1000 collisions.

The speed of a particle after collision with the wall $\tilde{V}$, can be written in the form

$$
\tilde{V}(\alpha, \theta, t, V)=V+\zeta(\alpha, \theta, t, V)
$$

where $V$ is the speed just before the collision. From this, the mean speed of an ensemble of particles just after the $n$-th collision takes the form

$$
\bar{V}_{n+1}=\bar{V}_{n}+\delta \bar{V}_{n}
$$

where

$$
\begin{aligned}
\bar{V}_{n}= & \int_{0}^{\infty} \int_{0}^{2 \pi} \int_{0}^{2 \pi} \int_{0}^{\pi} V \mathcal{F}_{n}(\alpha, \theta, t, V) \mathrm{d} \alpha \mathrm{d} \theta \mathrm{d} t \mathrm{~d} V, \\
\delta \bar{V}_{n}= & \int_{0}^{\infty} \int_{0}^{2 \pi} \int_{0}^{2 \pi} \int_{0}^{\pi} \zeta(\alpha, \theta, t, V) \mathcal{F}_{n}(\alpha, \theta, t, V) \\
& \times \mathrm{d} \alpha \mathrm{d} \theta \mathrm{d} t \mathrm{~d} V,
\end{aligned}
$$

and $\mathcal{F}_{n}(\alpha, \theta, t, V)$, is the phase space distribution function just before collision $n$. In the case of interest the distribution function can be factored in the form

$$
\mathcal{F}_{n}(\alpha, \theta, t, V)=F(\theta, \alpha) \rho_{V}(t) \rho_{n}(V),
$$

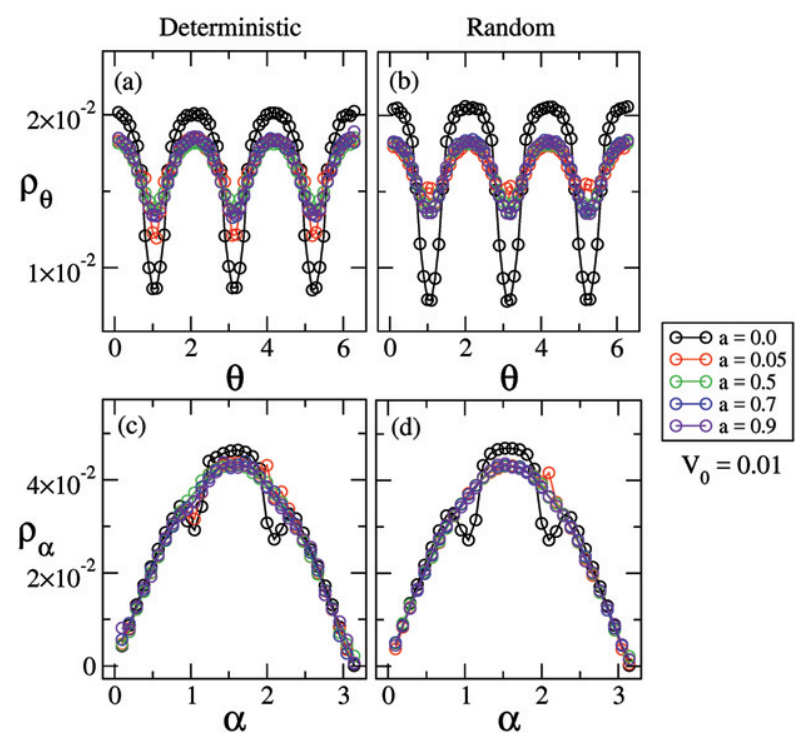

Fig. 4: (Color online) Plot of the numerical distribution functions $\rho_{\theta}(\theta)=\int \tilde{F}(\theta, \alpha) \mathrm{d} \alpha$ and $\rho_{\alpha}(\alpha)=\int \tilde{F}(\theta, \alpha) \mathrm{d} \theta$ for deterministic and non-deterministic (random) billiards at various amplitude of oscillations. The distributions are mostly determined by the geometry of the static billiard $(a=0)$, and are weakly modified by the wall oscillation. The inhomogeneous nature of these distributions are due to the presence of low period saddles in the phase space of the static oval billiard, where individual orbits spend longer times. The control parameters are $\epsilon=0.08$ and $p=3$.

where $F(\alpha, \theta)$ is the $\alpha-\theta$ distribution, $\rho_{V}(t)$ is the collision time distribution and $\rho_{n}(V)$ is the speed distribution function. The $F(\alpha, \theta)$ distribution is mainly determined by the billiard geometry and is only weakly modified by the wall oscillation, consequently it can be regarded as independent of the index $n$, the collision time distribution $\rho_{V}(t)$ depends on the speed but not on the index $n$ and the speed distribution function $\rho_{n}(V)$ depends on both the speed and the index. To understand the evolution of the mean speed of an ensemble we do not require to determine the evolution of the global distribution function. Inserting eq. (7) in eq. (5), and defining the partial mean

$U(V)=\int_{0}^{2 \pi} \int_{0}^{2 \pi} \int_{0}^{\pi} \zeta(\alpha, \theta, t, V) F(\theta, \alpha) \rho_{V}(t) \mathrm{d} \alpha \mathrm{d} \theta \mathrm{d} t$

we obtain a compact expression for the change in the mean speed

$$
\delta \bar{V}_{n}=\int_{0}^{\infty} \rho_{n}(V) U(V) \mathrm{d} V .
$$

Now, consider a second-order expansion of the partial mean $U(V)$ around the mean speed $\bar{V}_{n}$, of the distribution $\rho_{n}(V)$

$U(V) \approx U\left(\bar{V}_{n}\right)+U^{\prime}\left(\bar{V}_{n}\right)\left(V-\bar{V}_{n}\right)+\frac{1}{2} U^{\prime \prime}\left(\bar{V}_{n}\right)\left(V-\bar{V}_{n}\right)^{2}$. 
Replacing this expression of $U(V)$ in eq. (9) we obtain a second-order approximation of the change in the average speed

$$
\delta \bar{V}_{n}=U\left(\bar{V}_{n}\right)+\frac{1}{2} U^{\prime \prime}\left(\bar{V}_{n}\right)\left({\overline{V_{n}^{2}}}_{-\bar{V}_{n}}^{2}\right)
$$

The approximation in eq. (10) becomes poor as we move far from the distribution mean. However, the integrand in eq. (9) contributes less where the Taylor expansion of $U(V)$ is not accurate, because the speed distribution $\rho_{n}(V)$ drops for large and small values of $V$. However, this argument, becomes weaker when the speed distribution evolves in time to become wider, as observed in fig. 3 . Consequently, the precision of this approximation depends on the particular form of $U(V)$.

In the appendix of this manuscript, it is shown that, for thermal speed distributions [15] (similar to those in fig. 3), the relative error of eq. (11) is smaller than an upper bound independent of the mean speed. It is also shown that the upper bound is small for functions $U(V)$ falling slower than $1 / V$ or growing slower than $V^{3}$. Which is satisfied for the functions involved in the following calculations.

Replacing eq. (11) in eq. (4) we obtain a second-order approximation of the $n+1$ mean speed

$$
\bar{V}_{n+1}=\bar{V}_{n}+U\left(\bar{V}_{n}\right)+\frac{1}{2} U^{\prime \prime}\left(\bar{V}_{n}\right)\left({\overline{V_{n}^{2}}}_{-\bar{V}_{n}}^{2}\right)
$$

which depends on the mean of the quadratic speed at collision $n$. An equation for the evolution of the quadratic mean is also needed. For the quadratic speed after collision $\tilde{V}^{2}$, the collision rule can be also written in the form

$$
\tilde{V}^{2}(\alpha, \theta, t, V)=V^{2}+\psi(\alpha, \theta, t, V),
$$

where $V$ is again the speed before collision. Reproducing the same arguments used for the mean speed we obtain

$$
{\overline{V^{2}}}_{n+1}={\overline{V^{2}}}_{n}+W\left(\bar{V}_{n}\right)+\frac{1}{2} W^{\prime \prime}\left(\bar{V}_{n}\right)\left({\overline{V_{n}^{2}}}_{-\bar{V}_{n}}^{2}\right),
$$

where

$$
W(V)=\int_{0}^{2 \pi} \int_{0}^{2 \pi} \int_{0}^{\pi} \psi(\alpha, \theta, t, V) F(\theta, \alpha) \rho_{V}(t) \mathrm{d} \alpha \mathrm{d} \theta \mathrm{d} t .
$$

As will be shown later the inhomogeneous nature of $F(\theta, \alpha)$ is a fundamental aspect of super diffusion. It can be related to the presence of low period saddles in the static billiard [16], where the collisions occur more often [17], leading to an increase in the distribution value. Figure 4 we show line-integrated profiles of $F(\alpha, \theta)$ for both deterministic and random oscillations [14] of the billiard boundary.

In the limit of high speeds or small boundary oscillations it can be shown that $\zeta(\alpha, \theta, t, V) \approx \psi(\alpha, \theta, t, V) / 2 V$, which upon integration in $\{\theta, \alpha, t\}$ leads to

$$
U(V) \approx W(V) / 2 V
$$

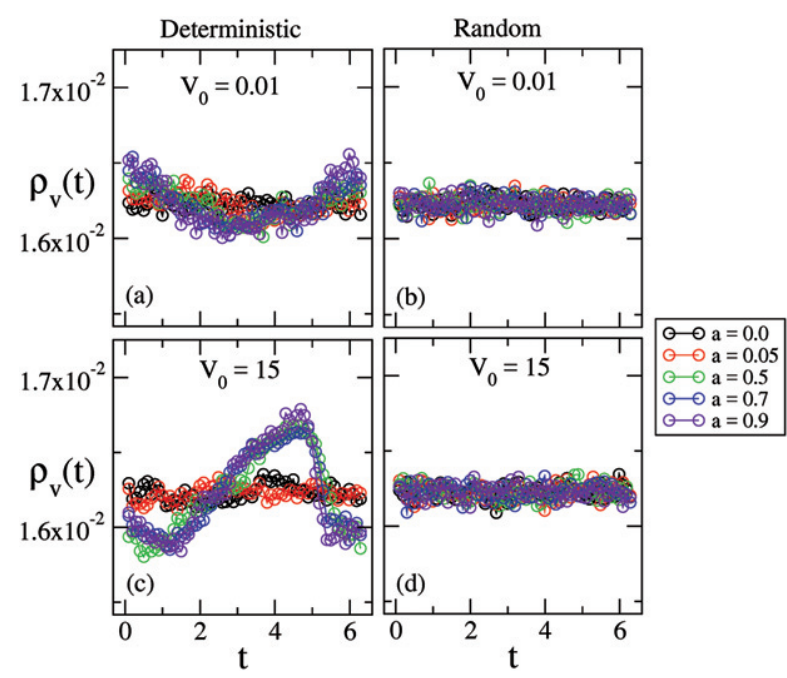

Fig. 5: (Color online) Plot of the numerical collision time distribution functions $\rho_{V}(t)$ for deterministic ((a), (c)) and non-deterministic (random) ((b), (d)) boundary oscillations at various amplitudes and two different initial speeds. For a low initial speed, both cases of the billiard initially have a similar behavior for the collision time distribution ((a), (b)) leading the systems to exhibit the normal diffusion. However, when the initial speed is high, the deterministic billiard exhibits a correlation between the subsequent collisions and consequently an inhomogeneous collision time distribution (c), while for the random billiard such correlation is not observed (d). The corresponding parameters are $\epsilon=0.08$ and $p=3$.

This results in an approximated form for the twodimensional mapping of the mean speed and the mean quadratic speed

$$
\begin{aligned}
\bar{V}_{n+1}= & \bar{V}_{n}+\frac{1}{2} W_{n} \overline{V_{n}^{2}} /{\overline{V_{n}}}^{3}+\frac{1}{2}\left(\frac{1}{2} \overline{V_{n}} W_{n}^{\prime \prime}-W_{n}^{\prime}\right) \\
& \times\left(\overline{V_{n}^{2}} /{\overline{V_{n}}}^{2}-1\right), \\
{\overline{V^{2}}}_{n+1}= & \bar{V}_{n}^{2}+W_{n}+\frac{1}{2} W_{n}^{\prime \prime}\left(\overline{V_{n}^{2}}-{\overline{V_{n}}}^{2}\right),
\end{aligned}
$$

where $W_{n}=W\left(\overline{V_{n}}\right), W_{n}^{\prime}=W^{\prime}\left(\overline{V_{n}}\right)$ and $W_{n}^{\prime \prime}=W^{\prime \prime}\left(\overline{V_{n}}\right)$. This mapping is general and its behavior depends on the particular form of the function $W(V)$ for the system under consideration. For instance, for the time-dependent oval billiard one can show that

$$
\begin{aligned}
\psi(\alpha, \theta, t)= & 4(a \epsilon)^{2} \cos ^{2}(p \theta) \sin ^{2}(t) \\
& -4 a \epsilon V \cos (p \theta) \sin (\alpha) \sin (t),
\end{aligned}
$$

and the collision time distribution can be approximated by (see fig. $5(\mathrm{a}),(\mathrm{c})$ )

$$
\rho_{V}(t)=\frac{1}{2 \pi}[1-a \epsilon \chi(V) \sin (t)]
$$

where $\chi(V)$ is a slowly changing function of $V$ that vanishes for $V=0$ and saturates at $\chi^{*}$ for large $V$. This distribution develops due to the correlation between subsequent 
collisions for higher speeds for which the time between collisions is small compared to the wall oscillation period. Expectedly, the harmonic part is removed when the wall oscillations are random (see fig. $5(\mathrm{~b}),(\mathrm{d}))$.

Inserting $\psi(\alpha, \theta, t)$ and $\rho_{V}(t)$ in eq. (15) and defining the following constants:

$$
\begin{aligned}
& \eta_{1}=(a \epsilon)^{2} \int_{0}^{\pi} \int_{0}^{2 \pi} F(\alpha, \theta) \sin (\alpha) \cos (p \theta) \mathrm{d} \theta \mathrm{d} \alpha \\
& \eta_{2}=(a \epsilon)^{2} \int_{0}^{\pi} \int_{0}^{2 \pi} F(\alpha, \theta) \cos ^{2}(p \theta) \mathrm{d} \theta \mathrm{d} \alpha
\end{aligned}
$$

we obtain

$$
W(V)=2 \eta_{2}+2 \eta_{1} \chi(V) V
$$

which inserted in the mappings for the average speed and the average quadratic speed (17), results in two coupled difference equations

$$
\begin{aligned}
& \overline{V_{n+1}}-\overline{V_{n}}=\eta_{1} \chi_{n}+\eta_{2} \overline{V_{n}^{2}} /{\overline{V_{n}}}^{3}, \\
& \overline{V_{n+1}^{2}}-\overline{V_{n}^{2}}=2 \eta_{2}+2 \eta_{1} \chi_{n} \overline{V_{n}}
\end{aligned}
$$

where $\chi_{n}=\chi\left(\overline{V_{n}}\right)$, and it was used that $\chi(V)$ changes slowly with the speed. More specifically the system satisfies $\chi^{\prime}\left(\overline{V_{n}}\right) \overline{V_{n}} \ll \chi\left(\overline{V_{n}}\right)$. These coupled difference equations can be solved asymptotically for small and large $n$ to give us a picture of the different diffusion regimes exhibited by the system during its evolution.

If the ensemble of particles begins with small speeds, i.e., of the order $a \epsilon$, we can use $\chi_{n} \rightarrow 0$, and the system can be integrated by taking the continuous limit $\bar{f}_{n}-$ $\bar{f}_{n-1} \approx d f(n) / d n$, which results in

$$
\overline{V_{s}^{2}}(n)=V_{0}^{2}+2 \eta_{2} n
$$

where the subscript $s$ indicates small $n$. This solution can be replaced in the mean speed difference equation to obtain an approximated solution for the mean speed valid for few collisions

$$
\overline{V_{s}}(n)=\left(V_{0}^{2}+2 \eta_{2} n\right)^{1 / 2} .
$$

Notice that this solution emerged after assuming an homogeneous phase distribution, i.e., $\chi_{n} \rightarrow 0$, which is also appropriate when the collision phase with the wall is random. As the mean speed of the ensemble grows by normal diffusion, the time between consecutive collisions is reduced and the collision time distribution becomes inhomogeneous due to the coherent acceleration and deceleration of individual particles (cf. fig. 2, panel B). Leading naturally to a situation where $\chi_{n} \neq 0$.

In the new regime, the value of $\chi_{n}$ saturates to $\chi^{*}$ when large speeds are achieved. Then, provided that $\overline{V_{n}^{2}}$ and ${\overline{V_{n}}}^{2}$ are of the same order, we have that ${\overline{V_{n}^{2}}}_{\overline{V_{n}}}^{3} \rightarrow 0$ for large $\overline{V_{n}}$. The last statement is valid for unimodal distributions, like the Maxwell-Boltzmann one [15] for which $\overline{V_{n}^{2}} /{\overline{V_{n}}}^{3}=4 /(\pi \bar{V})$, but is also valid for delocalized situations, like the uniform distribution for which
${\overline{V_{n}^{2}}}^{2}{\overline{V_{n}}}^{3}=4 /(3 \bar{V})$. This guarantees that the ratio $\overline{V_{n}^{2}} /{\overline{V_{n}}}^{3}$ goes to zero as the mean velocity grows regardless of how localized is the speed distribution.

From this, in the deterministic high velocity limit, eq. (23) becomes

$$
\overline{V_{n+1}}-\overline{V_{n}} \approx \eta_{1} \chi^{*}
$$

which can be integrated to obtain the evolution rule for the high speeds regime

$$
\overline{V_{l}}(n)=V_{0}+\eta_{1} \chi^{*} n .
$$

Here, the subscript $l$ indicates large $n$. For regular values of $\eta_{1}, \eta_{2}$ and $\chi^{*}$, the difference $V_{l}(n)-V_{0}$ is small compared to $V_{s}(n)-V_{0}$ for small $n$, while the opposite happens for large $n$. Consequently, we can combine eq. (26) and eq. (28) to obtain a single approximate solution valid for all stages of the ensemble evolution

$$
\bar{V}(n)=\left(V_{0}^{2}+2 \eta_{2} n\right)^{1 / 2}+\eta_{1} \chi^{*} n .
$$

An interesting feature of this solution is that $\eta_{1}$ vanishes if $F(\alpha, \theta)$ is homogeneous, so that, a deterministic billiard without low period saddles in phase space will only exhibit normal diffusion because of the uniform distribution of particles in the phase space. The combined solution eq. (29), corresponds to the continuous lines in fig. 2 in excellent agreement with the numerical simulations for all the ensembles considered.

As a short summary, in this letter we have shown that an inhomogeneous particle distribution function on the phase space of the static billiard leads to the development of anomalous diffusion regimes in time-dependent situations, and for the particular case of oval billiards, explains the transitions from normal to super diffusion. These results were obtained by identifying the asymptotic dynamics of a two-dimensional mapping for the first and second moments of the speed distribution. The presented treatment, is sufficiently general to study other anomalous diffusion regimes, diverse billiard shapes and more general mappings.

$$
* * *
$$

$\mathrm{MH}$ thanks CAPES for financial support. DC, ILC and EDL thank CNPq (433671/2016-5, 300632/2010-0 and 303707/2015-1) and ILC and EDL thank the São Paulo Research Foundation (FAPESP) (2011/19296-1 and 2017/14414-2) for their financial support.

\section{Appendix}

In this section we are interested in providing a more compeling physical argument for the validity of the approximations used in eq. (11) and eq. (14). First of all consider a large collection of non-interacting particles confined in a two-dimensional region in equilibrium with a thermal bath. 
The probability of a particle having a velocity $\vec{V}=\left(V_{x}, V_{y}\right)$ is proportional to the Boltzmann Factor $\exp \left[-\frac{m}{2 k_{B} T}\left(V_{x}^{2}+V_{y}^{2}\right)\right]$, from which, the probability of having a speed $V$, with arbitrary direction is given by

$$
\rho_{M B}(\bar{V}, V)=\frac{\pi}{2 \bar{V}^{2}} V \exp \left[-\frac{\pi}{4}\left(\frac{V}{\bar{V}}\right)^{2}\right] .
$$

Here, the mean speed $\bar{V}=\sqrt{\pi k_{B} T / 2 m}$ was used to characterize the speed distribution instead of the temperature. This distribution has very similar features to those observed in the numerical simulations (fig. 3), but corresponds to a system in thermodynamic equilibrium, which is not our case. However we can consider that our system is approximately described as a sequence of equilibrium configurations, where each collision of the ensemble with the moving boundary delivers a bit of energy to the ensemble, changing its temperature (or mean speed in our case).

Now, let us consider a given function of the speed with the form $U_{\lambda}(V)=V^{\lambda}$, with $\lambda$ some real number. The mean value of this function under $\rho_{M B}$ can be obtained by direct integration and has the form

$$
\begin{aligned}
\bar{U}_{\lambda}(\bar{V}) & =\int_{0}^{\infty} \rho_{M B}(\bar{V}, V) V^{\lambda} \mathrm{d} V \\
& =\lambda 2^{\lambda-1} \pi^{-\lambda / 2} \Gamma(\lambda / 2) \bar{V}^{\lambda}=c_{\lambda} \bar{V}^{\lambda} .
\end{aligned}
$$

Now, let us approximate the value of $\bar{U}_{\lambda}$ with the secondorder expansion $\widetilde{U}_{\lambda}$, obtained by expanding $U_{\lambda}(V)$ about the mean of the distribution, which dispenses the integration step, i.e.,

$$
\widetilde{U}_{\lambda}(\bar{V})=U_{\lambda}(\bar{V})+\frac{1}{2} U_{\lambda}^{\prime \prime}(\bar{V})\left(\overline{V^{2}}-\bar{V}^{2}\right) .
$$

Here, we can use $\left(\overline{V^{2}}-\bar{V}^{2}\right)=(4 / \pi-1) \bar{V}^{2}$, valid for the Maxwell-Boltzmann distribution and $U_{\lambda}^{\prime \prime}(V)=\lambda(\lambda-1)$ $V^{\lambda-2}$, so that we have the approximated solution

$$
\widetilde{U}_{\lambda}(\bar{V})=\left(1+\frac{1}{2 \pi} \lambda(\lambda-1)(4-\pi)\right) \bar{V}^{\lambda}=c_{\lambda}^{\prime} \bar{V}^{\lambda} .
$$

Notice that eq. (A.2) and eq. (A.4) have the same dependence in $\bar{V}$, then, the relative error of the approximation $\varepsilon_{\lambda}=|\bar{U}(\bar{V})-\widetilde{U}(\bar{V})| /|\bar{U}(\bar{V})|$ is independent of the mean speed of the distribution. Namely, $\varepsilon_{\lambda}$ takes the form

$$
\varepsilon_{\lambda}=\frac{\left|c_{\lambda}-c_{\lambda}^{\prime}\right|}{\left|c_{\lambda}\right|}
$$

where the coefficients $c_{\lambda}$ and $c_{\lambda}^{\prime}$ are independent of $\bar{V}$. Figure 6 , we show the relative error of $\widetilde{U}_{\lambda}$ for $-1<\lambda<3$, under a Maxwell-Boltzmann distribution. The difference is below $1 \%$ for $-0.2<\lambda<2.4$, and grows up to $\sim 10 \%$ at $\lambda=-1$ and $\lambda=3$.

This result suggests that, for speed distributions with thermal characteristics, the second-order approximation

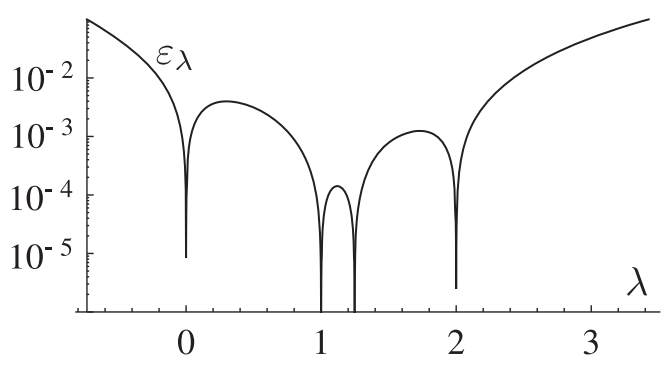

Fig. 6: Relative error of $\widetilde{U}_{\lambda}(\bar{V})$ for $-1<\lambda<3$. Outside this domain the relative error grows above $10 \%$.

presented in eq. (11) is appropriate if the function $U(V)$ grows or decays slowly with the speed $V$. For the context of this work, the previous analysis can be extended to functions of the form

$$
U(V)=\sum_{i=0}^{N} a_{i} U_{\lambda_{i}}(V)=\sum_{i=0}^{N} a_{i} V^{\lambda_{i}},
$$

where $\left\{\lambda_{i}\right\}_{i=1}^{N}$ is a set of real numbers. For this function we have the exact and approximated means

$$
\bar{U}(\bar{V})=\sum_{i=0}^{N} a_{i} c_{\lambda_{i}} \bar{V}^{\lambda_{i}}, \quad \widetilde{U}(\bar{V})=\sum_{i=0}^{N} a_{i} c_{\lambda_{i}}^{\prime} \bar{V}^{\lambda_{i}},
$$

with $c_{\lambda_{i}}$ and $c_{\lambda_{i}}^{\prime}$ given by eq. (A.2) and eq. (A.4). Here, the Cauchy-Schwarz inequality ensures that

$$
|\bar{U}(\bar{V})-\widetilde{U}(\bar{V})| \leq\left|\sum_{i=0}^{N} \frac{c_{\lambda_{i}}-c_{\lambda_{i}}^{\prime}}{c_{\lambda_{i}}}\right|\left|\sum_{j=0}^{N} a_{j} c_{\lambda_{j}} V^{\lambda_{j}}\right|
$$

Dividing both sides by $|\bar{U}(\bar{V})|$ and using the triangle inequality in the r.h.s., we obtain an upper bound for the approximation error of the function $\widetilde{U}(\bar{V})$,

$$
\varepsilon[\widetilde{U}]=\frac{|\bar{U}(\bar{V})-\widetilde{U}(\bar{V})|}{|\bar{U}(\bar{V})|} \leq \sum_{i=0}^{N} \varepsilon_{\lambda_{i}}
$$

where the $\varepsilon_{\lambda_{i}}$ are as given in eq. (A.5) and deppend on $\lambda_{i}$ as depicted in fig. 6. Consequently, the upper bound of the approximation error of $\widetilde{U}(\bar{V})$ is independent of the mean speed $\bar{V}$, which guarantees the stability of the approximated mapping in eq. (17) for speed distributions with thermal characteristics.

\section{REFERENCES}

[1] Fermi E., Phys. Rev., 75 (1949) 1169.

[2] Chernov N. and Markarian R., Chaotic Billiards (American Mathematical Society) 2006.

[3] Berry M. V., Eur. J. Phys., 2 (1981) 91.

[4] Sinai YA. G., Russ. Math. Surv., 25 (1970) 137.

[5] Lenz F., Diakonos F. K. and Schmelcher P., Phys. Rev. Lett., 100 (2008) 014103. 
[6] Leonel E. D. and Bunimovich L. A., Phys. Rev. Lett., 104 (2010) 224101.

[7] Loskutov A., Ryabov A. B. and Akinshin L. G., J. Phys. A: Math. Gen., 33 (2000) 7973.

[8] Gelfreich V. and Turaev D., J. Phys. A: Math. Theor., 41 (2008) 212003.

[9] Gelfreich V., Rom-Kedar V. and Turaev D., Chaos, 22 (2012) 033116.

[10] Oliveira D. F. M., Silva M. R. and Leonel E. D., Physica A, 436 (2015) 909.

[11] Leonel E. D., Oliveira D. F. M. and Loskutov A., Chaos, 19 (2009) 033142.
[12] Batistić B. and Robnik M., J. Phys. A, 44 (2011) 365101.

[13] Batistić B., Phys. Rev. E., 90 (2014) 032909.

[14] Leonel E. D., Galia M. V. C., Barreiro L. A. and Oliveira D. F. M., Phys. Rev. E, 94 (2016) 062211.

[15] ReIF F., Fundamentals of Statistical and Thermal Physics (Waveland Press) 2009.

[16] Hansen M., de Carvalho R. E. and Leonel E. D., Phys. Lett. A, 380 (2016) 3634.

[17] Hansen M., da Costa D. R., Caldas I. L. and Leonel E. D., Chaos, Solitons Fractals, 106 (2018) 355. 